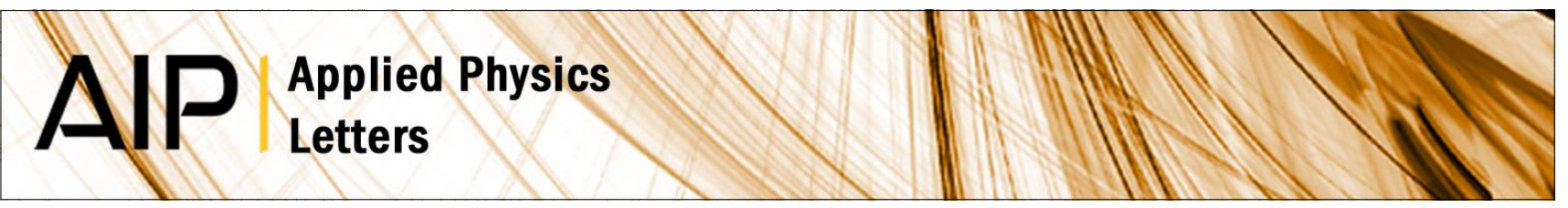

\title{
Experimental study of broadband unidirectional splitting in photonic crystal gratings with broken structural symmetry
}

Evrim Colak, Andriy E. Serebryannikov, A. Ozgur Cakmak, and Ekmel Ozbay

Citation: Appl. Phys. Lett. 102, 151105 (2013); doi: 10.1063/1.4800147

View online: http://dx.doi.org/10.1063/1.4800147

View Table of Contents: http://apl.aip.org/resource/1/APPLAB/v102/i15

Published by the American Institute of Physics.

Additional information on Appl. Phys. Lett.

Journal Homepage: http://apl.aip.org/

Journal Information: http://apl.aip.org/about/about_the_journal

Top downloads: http://apl.aip.org/features/most_downloaded

Information for Authors: http://apl.aip.org/authors

\section{ADVERTISEMENT}
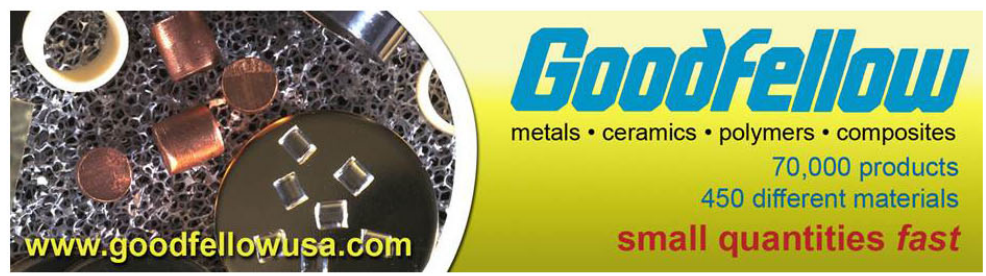


\title{
Experimental study of broadband unidirectional splitting in photonic crystal gratings with broken structural symmetry
}

\author{
Evrim Colak, ${ }^{1}$ Andriy E. Serebryannikov, ${ }^{2}$ A. Ozgur Cakmak, ${ }^{1, a)}$ and Ekmel Ozbay ${ }^{1}$ \\ ${ }^{1}$ Nanotechnology Research Center, Bilkent University, 06800 Ankara, Turkey \\ ${ }^{2}$ Hamburg University of Technology, E-3, D-21071 Hamburg, Germany
}

(Received 2 November 2012; accepted 21 March 2013; published online 15 April 2013)

\begin{abstract}
It is experimentally demonstrated that the combination of diode and splitter functions can be realized in one broadband reciprocal device. The suggested performance is based on the dielectric photonic crystal grating whose structural symmetry is broken owing to non-deep corrugations placed at one of the two interfaces. The study has been performed at a normally incident beam-type illumination obtained from a microwave horn antenna. The two unidirectionally transmitted, deflected beams can show large magnitude and high contrast, while the angular distance between their maxima is $90^{\circ}$ and larger. The dual-band unidirectional splitting is possible when using TM and TE polarizations. (C) 2013 American Institute of Physics. [http://dx.doi.org/10.1063/1.4800147]
\end{abstract}

During the last years, there has been a growing interest in asymmetric transmission, a phenomenon which manifests itself in the strong contrast between the forward and the backward transmission in the structures that contain only linear isotropic constituents and, thus, are reciprocal in sense of the Lorentz reciprocity principle. ${ }^{1-5}$ It can appear in structures with the broken structural symmetry. The strong redistribution of the transmitted wave energy is managed either in favor of the higher diffraction orders, as in photonic crystal (PC) gratings, ${ }^{1-3}$ or the cross polarization, as in structures based on subwavelength resonators. ${ }^{6,7}$ Asymmetric transmission can be used to emulate the diode function simultaneously with another, e.g., bending function in one device. ${ }^{1,3}$ Clearly, the classical (nonreciprocal) diode function cannot be extracted from the resulting mechanism.

Finite-thickness PC based transmission gratings that are structurally asymmetric due to the one-side corrugations have been recently suggested ${ }^{8}$ and then extensively studied with the focus on asymmetric transmission. ${ }^{1-4,9-11}$ In these structures, the dominant mechanism is based on the merging of the effects of dispersion of the Floquet-Bloch (FB) modes of the PC and asymmetric diffraction and coupling at the interfaces. The higher orders may take all the incident-wave energy in the forward case, while the transmission is blocked in the backward case. ${ }^{3}$ Two basic broadband regimes, i.e., unidirectional single-beam deflection (angle of incidence $\theta \neq 0$ ) and unidirectional two-beam splitting $(\theta=0)$, have been distinguished. ${ }^{1,3}$ Achieving a reasonable compromise between the efficiency and bandwidth remains as a big problem, especially at a realistic beam-type illumination. One more class of the asymmetric structures presents stacks of prisms based on finite-size pieces of PCs. ${ }^{5,12-14}$ A PC grating with deep corrugations can be considered as a structure that consists of such prisms which are periodically placed at the top of the noncorrugated slab of PC. ${ }^{1,2,4,10}$ Yet, the simplest one-side corrugations that are obtained by removing every second rod from

\footnotetext{
${ }^{\text {a) }}$ Author to whom correspondence should be addressed. Electronic mail: atilla@ee.bilkent.edu.tr.
}

an interface layer of the noncorrugated slab of PC can be most advantageous. ${ }^{3}$

In this paper, we experimentally demonstrate that the broadband unidirectional splitting can be obtained in a PC grating at beam-type illumination created by the microwave horn antenna. The width and magnitude of the outgoing beams and the angular distance between them can be controlled by variations in frequency and/or angle of incidence. In contrast to the earlier works, both TM and TE polarizations will be studied, for which either electric field vector or magnetic field vector is parallel to the rods, respectively. The considered regimes of asymmetric transmission will include those whose features cannot be predicted by using wave vector diagrams. The PC grating has been designed based on the compromise between the bandwidth and efficiency. Following our recent experience, we utilize non-deep one-side corrugations which are obtained by removing some rods from one of the two interface layers of a noncorrugated slab of PC. The used PC grating is similar to those in Ref. 3 but has a more suitable value of the rod-diameter-to-lattice-constant ratio, $d / a$. Note that another combined regime, i.e., single-beam unidirectional deflection (bending), has been experimentally studied in Ref. 2. Throughout the paper, $\rightarrow$ denotes the forward-transmission case (corrugated-side illumination, or $U$-case) and $\leftarrow$ denotes the backward-transmission case (noncorrugated-side illumination, or $L$-case).

According to Refs. 1 and 3, the unidirectional splitting is a wideband operation regime realizable at $\theta=0$ and the linearly polarized plane incident waves, for which $T^{\rightarrow}$ $=T_{\Sigma}^{\vec{C}}=\sum_{(n)} t_{-n}^{\rightarrow}+t_{+n}^{\rightarrow}$ and $T^{\leftarrow}=T_{\Sigma}^{\leftarrow} \approx 0$, where $T^{\rightarrow}$ and $T \leftarrow$ mean the forward and backward transmittances, respectively. In turn, $t_{+n}^{\rightarrow}$ and $t_{-n}^{\rightarrow}$ mean the $\pm n$ th-order forward partial transmittances. This regime requires such shapes of isofrequency dispersion contours (IFCs) of FB modes that there is no coupling to the waves which may propagate in the surrounding air half-spaces in the vicinity of zero tangential wave number $k_{x}$ [ $x$ axis is assumed to be along the interfaces, as in Fig. 1(a)]; see Ref. 1. Thus, zero order must be uncoupled in the vicinity of $\theta=0$. However, the coupling can be possible at the corrugated-side illumination in a certain range of $\left|k_{x}\right| \neq 0$ for the higher diffraction orders, which 

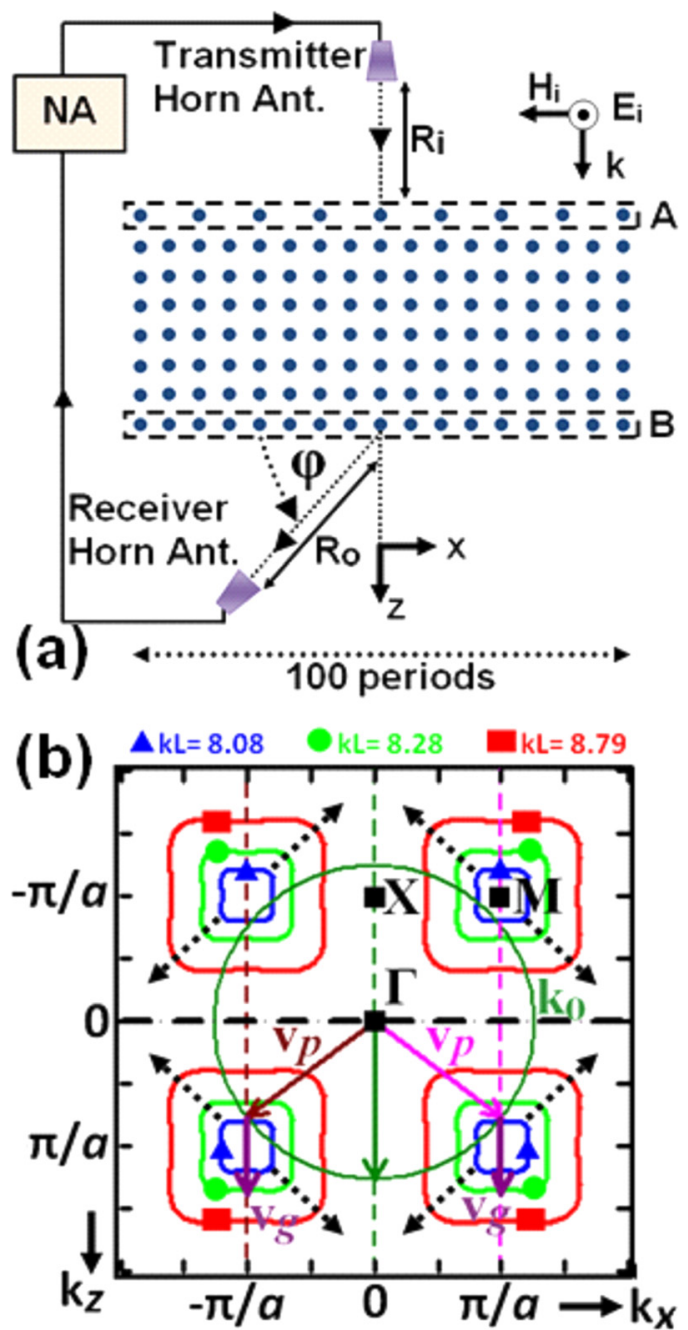

FIG. 1. (a) Schematic of the experimental setup; the $U$-case is depicted; for the $L$-case, layers $A$ and $B$ are replaced with each other. (b) The IFCs of PC at the third band, TM polarization: blue lines and triangles $-k L=8.08$ $(18.67 \mathrm{GHz})$, light green lines and filled circles- $k L=8.28(19.14 \mathrm{GHz})$, red lines and rectangles $-k L=8.79(20.3 \mathrm{GHz})$; the dashed arrowspossible directions of the group velocity $\left(v_{g}\right)$; the input interface is assumed to be parallel to the black dashed line; solid green circle- the IFC for air at $k L=8.08$; vertical dashed pink, green, and brown lines-the +1 st, 0 th, and -1 st order construction lines, respectively; solid green arrow-the incident wave vector $\left(k_{0}\right)$, solid pink and brown arrows-directions of the phase velocities of the outgoing beams $\left(v_{p}\right)$, and solid purple arrows-direction of the group velocity inside the PC, all at $k L=8.08$.

may propagate due to the corrugations but cannot appear at the noncorrugated interface. Hence, the IFCs must be centered around $\mathrm{M}$ point, while those around $\mathrm{X}$ or/and $\Gamma$ point are not appropriate. The situation can be obtained that the higher orders contribute to $T^{\rightarrow}$, but there is no coupling at all if the noncorrugated side is illuminated, so that $T^{\leftarrow}=0$. Note that the FB modes showing IFCs located around M point are well known from the spatial filtering ${ }^{15}$ and negative refraction ${ }^{16}$ studies.

For the purposes of the experimental study, we designed a PC grating that is based on a square-lattice, two-dimensional, dielectric PC, which is composed of circular alumina rods with a relative permittivity $\varepsilon=9.61$ and diameter $d=3.1 \mathrm{~mm}$. The lattice constant is $a=10.33 \mathrm{~mm}$, so that $d / a=0.3$. The PC grating is assembled as an $12 \times 100$ array. Extensive simulations have been carried out in order to determine the optimum design parameters, which would enable a reasonable compromise between the transmission efficiency and bandwidth. The corrugations are obtained by removing every second rod from one of the two interface layers of a noncorrugated slab of PC. The schematics of the PC grating and experimental setup are shown in Fig. 1(a). The setup contains an Anritsu two-port 37369A Network Analyzer and two standard pyramidal K-band horn antennas. In the experiment, the incidence distance is $R_{i}=25 \mathrm{~cm}$, which corresponds to an approximate spot size of $12 a$ at $f=22 \mathrm{GHz}$. The receiver antenna scans a semicircular trajectory of radius $R_{O}=1 \mathrm{~m}$ so that observation angle $\varphi$ is varied. In $U$-case, layer $A$ (nonregular layer that plays the role of corrugation) is placed on the transmitter antenna side, while layer $B$ (regular, i.e., noncorrugated interface layer) is at the exit side, as depicted in Fig. 1(a). In $L$-case, layer $A$ is located at the exit side.

The design has been based on the analysis of IFCs for the corresponding infinite PC. An example is presented in Fig. 1(b) for TM-polarization. The $k L$-values in Fig. 1(b) are taken from the range, where unidirectional splitting is expected to appear. To provide connection with the general theory of the PC gratings, ${ }^{1}$ we also calculated the transmittance at plane-wave illumination (see Fig. 2(a)). Now, the structure is assumed to be infinite along the $x$ axis. In the corresponding $L$-case, transmission vanishes in the ranges labeled by " 1 " and " 2. ." The first range of the unidirectional splitting (denoted by " 1 ") is wider. It shows $17.9 \%$ bandwidth. The second one (denoted by "2") is characterized by a higher transmittance. It follows from the obtained results (not shown here) that the locations of these ranges can be strongly affected by variations in d/a. Simulations have also been performed at a Gaussian beam-type illumination [see

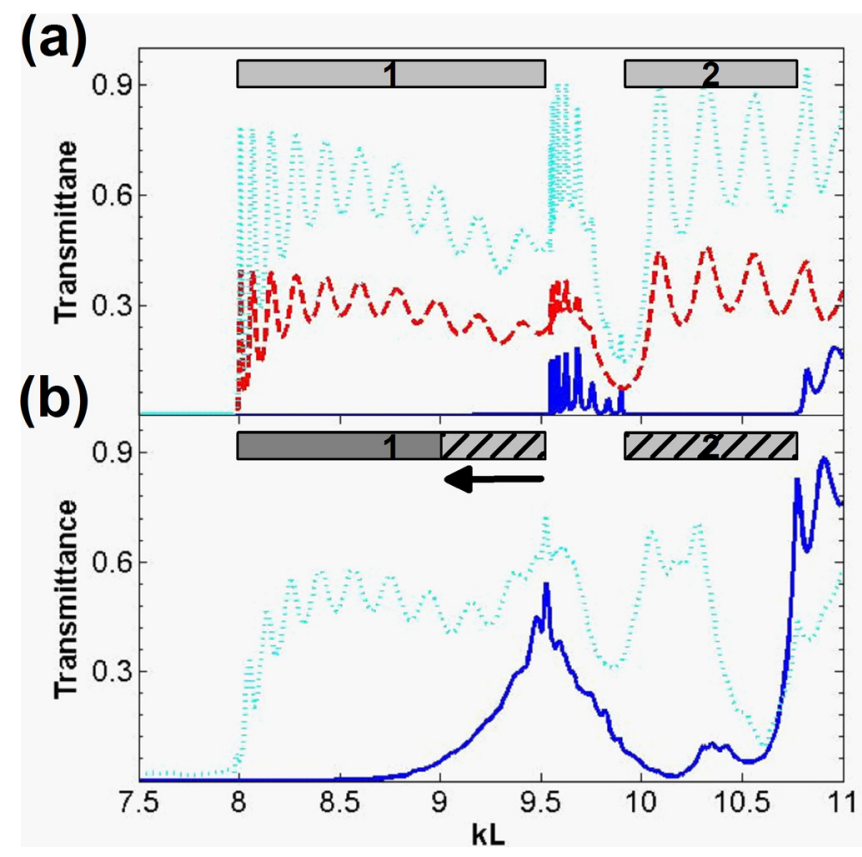

FIG. 2. Transmittance (TM polarization) as a function of $k L$ at (a) planewave illumination: solid dark blue line $-t_{0}$, dashed red line $-t_{ \pm 1}$, dotted cyan line $-T^{\rightarrow}$; gray rectangles labeled as " 1 " and " 2 "- -first and second ranges of unidirectional splitting; (b) Gaussian illumination: dotted cyan line $-T^{\rightarrow}$, solid dark blue line $-T^{\leftarrow}$; the shaded regions in gray rectangles show the vanishing unidirectionality. 
Fig. 2(b)]. The obtained results indicate that not all of the features can be predicted by the IFC and plane-wave transmission analyses. It is depicted that the first range of unidirectional splitting is shrunk and the upper edge is downshifted to lower frequencies as the arrow in Fig. 2(b) implies, while the second range is lost.

The experimental results for the electric field intensity at TM-polarization, $\tau_{\Sigma}$ and $\tau_{\Sigma}^{\leftarrow}$, are presented in Figs. 3(a) and 3 (b) on $(\varphi, f)$-plane. This way of the presentation allows us to directly observe the evolution of the directional (the difference between the forward and the backward transmissions) and directive (the outgoing beam width) features with the frequency variation. The structure is normally illuminated with a horn antenna, as shown in Fig. 1(a). Thus, the incident wave has a wide plane-wave angular spectrum. In Fig. 3, four typical frequency ranges can be distinguished, depending on the manner in which the asymmetric transmission manifests itself. They are denoted by A, B, C, and D. The asymmetry is strongest for range $\mathrm{A}$, which extends approximately from $18 \mathrm{GHz}$ to $21.1 \mathrm{GHz}$. It is associated with the unidirectional range which is labeled by "1" in Fig. 2. Here, the backward transmission tends to vanish in the entire range of $\varphi$ variation. Hence, the diode-like unidirectional splitting similar to that predicted at $\theta=0$ for the plane-wave illumination in Refs. 1 and 3 is obtained now for the beam-type incident wave. The unidirectional transmission co-exists here with splitting, while both of the outgoing beams are deflected wrt the incidence direction. The bigger part of this range possesses topologically the same IFCs as those in Fig. 1(b). The vicinity of $f=19 \mathrm{GHz}$ looks most preferable, because the electric field in the exit half-space is quite strong, while the separation between the outgoing beams in units of $\varphi$ is quite large.

Range B in Fig. 3 approximately corresponds to $21.1 \mathrm{GHz}$ $\leq f \leq 21.8 \mathrm{GHz}$. Therein, the transmission associated with zero order is possible in the vicinity of $\varphi=90^{\circ}$. In contrast to range $\mathrm{A}$, now the backward transmission does not vanish in the entire range of $\varphi$ variation, so that the exactly diode-like operation regime cannot be obtained.

According to Fig. 2(a) ( $k L$-range labeled by "2") and the dispersion results (not shown), one might expect that zero order is not coupled and, thus, unidirectional splitting also appears in range $\mathrm{C}(21.8 \mathrm{GHz} \leq f \leq 24.5 \mathrm{GHz})$. Indeed, as seen in Figs. 3(a) and 3(b), the zero order relevant transmission vanishes. However, the unidirectional transmission is partial in range $\mathrm{C}$, i.e., it occurs within a finite but not the entire range of $\varphi$ variation. In particular, transmission is nonzero only in $U$-case at $30^{\circ}<\varphi<60^{\circ}$ and $120^{\circ}<\varphi<150^{\circ}$. Strong backward transmission within narrow $\varphi$ ranges is observed in the horn antenna experiment [Fig. 3(b)], despite that such a situation does not appear at plane-wave illumination. The nature of this effect is not the focus of this study. Finally, in range $\mathrm{D}(24.5 \mathrm{GHz} \leq f \leq 26 \mathrm{GHz})$ the transmission associated with zero order again becomes significant. Here, asymmetry remains since $\tau_{\Sigma} \neq \tau_{\Sigma}^{\leftarrow}$ but $\tau_{\Sigma}^{\vec{D}} \neq 0$ and $\tau_{\Sigma}^{\leftarrow} \neq 0$, while $\tau_{\Sigma}^{\leftarrow}=0$ is required for the diode-like operation. To compare, the simulation results obtained under a Gaussian beam illumination are presented in Figs. 3(c) and 3(d). Most of the features of the transmitted electric field distributions agree very well with the experimental findings.

Figure 4 shows the measured electric field intensity vs $\varphi$ in $U$ and $L$ cases. The presented results are sampled from Figs. 3(a) and 3(b). The intensity values can be obtained at the maxima, for which max $\tau_{-1}+\max \tau_{+1}>\max \tau_{\text {inc }}$, where $\tau_{-1}, \tau_{+1}$, and $\tau_{\text {inc }}$ mean the intensity of the outgoing beams that are associated with the -1 st and +1 st diffraction orders and intensity of the incident beam, respectively. The diffraction angles, $\phi_{ \pm 1}$, measured in the counter-clockwise direction wrt the normal to the exit side, are given by the grating formula, $\sin \phi_{n}=\sin \theta+2 \pi n / k L$, in which we take $n= \pm 1$,
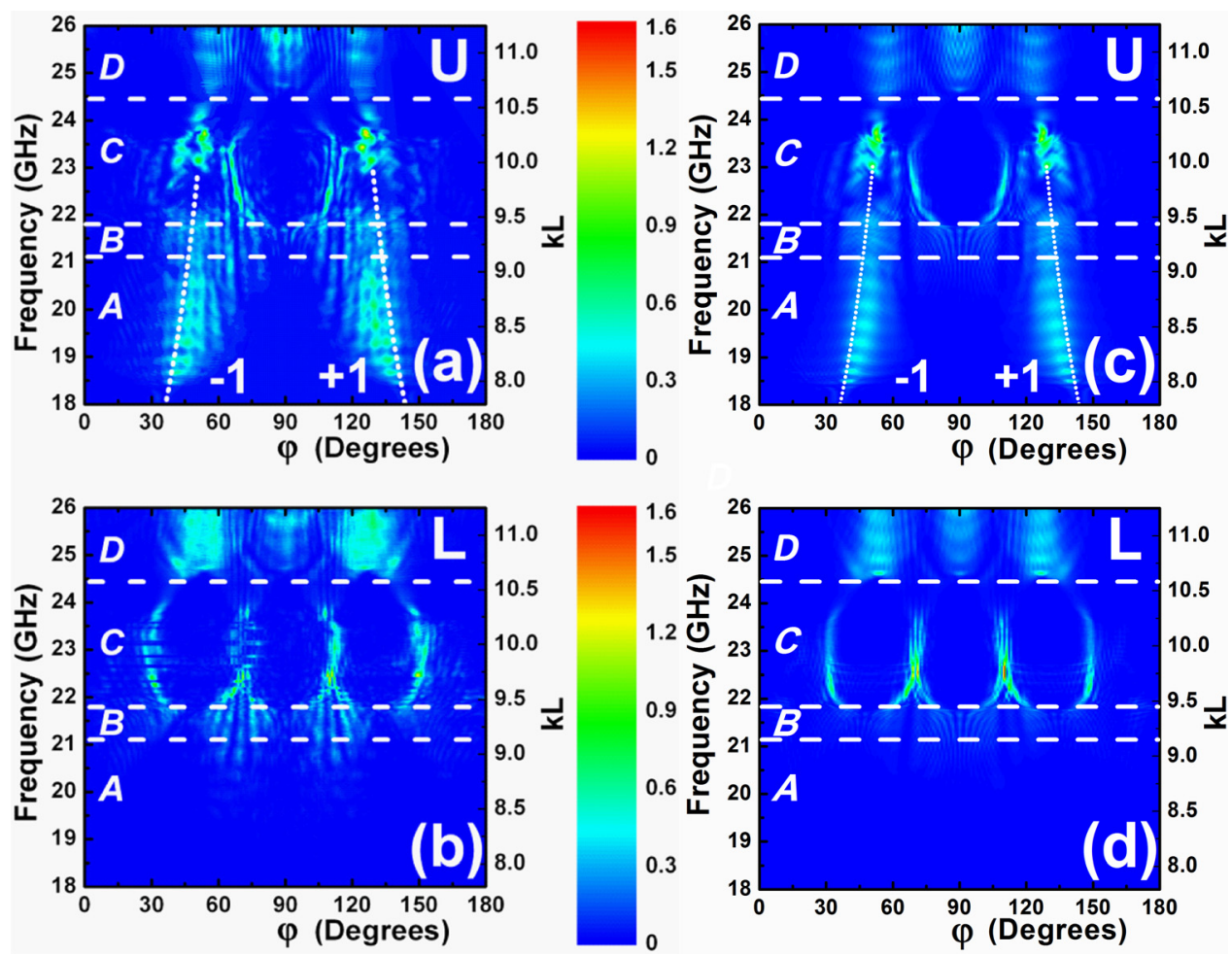

FIG. 3. Maps of the measured transmitted electric field intensity for (a) $U$-case and (b) $L$-case, and the calculated intensity at the Gaussian-beam illumination for (c) $U$-case and (d) $L$-case; TM polarization; white dashed lines show the approximate boundaries between ranges $\mathrm{A}$, $\mathrm{B}, \mathrm{C}$, and $\mathrm{D}$; white dotted lines show $\varphi=\varphi_{g}=\pi / 2+\phi_{ \pm 1}$ obtained using the grating formula. 

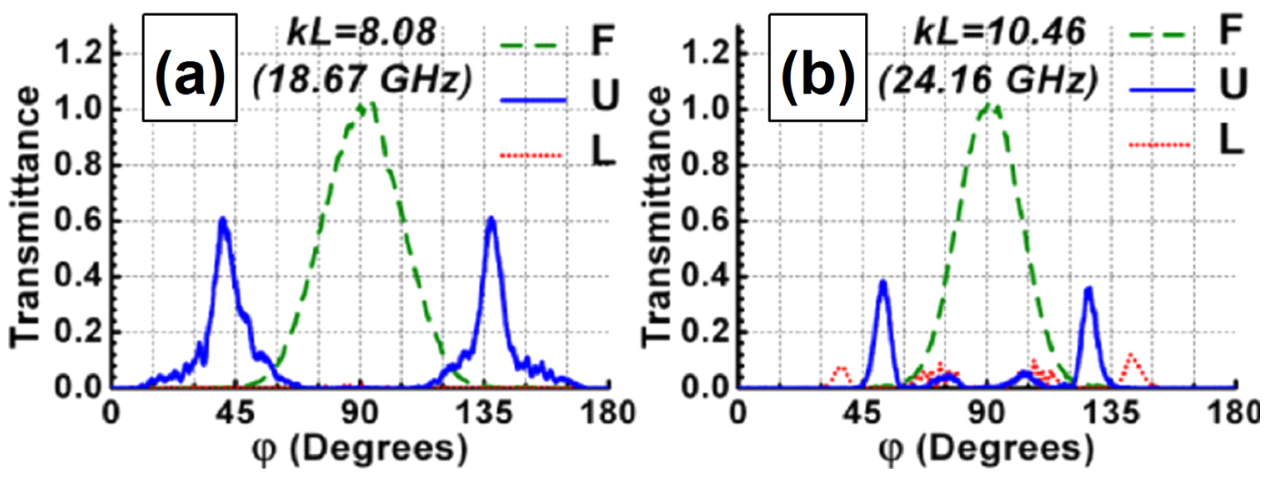

FIG. 4. The transmitted electric field intensity vs $\varphi$ at $k L=8.08$ (a) and 10.46 (b); solid dark blue line- $U$-case, dotted red line- $L$-case, dashed green line-free-space case (labeled as F); TM polarization.
$L=2 a$, and $\theta=0$. They are equal to $\pm 51^{\circ}$ and $\pm 36.2^{\circ}$ at $k L=8.08$ and 10.46, respectively, corresponding to the following pairs of the values of $\varphi=\varphi_{g}=\pi / 2+\phi_{ \pm 1}$ : $\left(141^{\circ}, 39^{\circ}\right)$ and $\left(126.2^{\circ}, 53.8^{\circ}\right)$. To compare, the maxima are observed in Fig. 4 ( $U$-case) for the following values of $\varphi=\varphi_{m}:\left(137.6^{\circ}, 40.3^{\circ}\right)$ and $\left(126.7^{\circ}, 52.5^{\circ}\right)$. The difference between $\varphi_{g}$ and $\varphi_{m}$ can be connected with the effect of plane-wave components of the beam angular spectrum, which correspond to $\theta \neq 0$ and are coupled to the FB wave at normal incidence of a finite beam. Additionally, the fabrication imperfections play a role.

Besides, Fig. 4(b) illustrates some peculiarities of the $\varphi$ dependence of the electric field intensity in range $\mathrm{C}$. Here, one-way transmission with $\tau_{\Sigma} \neq 0$ and $\tau_{\Sigma}^{\leftarrow} \approx 0$ and the maxima at $\varphi=52.4^{\circ}$ and $\varphi=127.4^{\circ}$ are observed. At the same time, the inverse regime, i.e., that with $\tau_{\Sigma} \approx 0$ and $\tau_{\Sigma}^{\leftarrow} \neq 0$ (Ref. 3) is obtained while the maxima of $\tau_{\Sigma}^{\leftarrow}$ are located at $\varphi=37.5^{\circ}$ and $\varphi=142^{\circ}$. Their locations have not been predicted by using the grating formula with $L=2 a$. Hence, in contrast to range $\mathrm{A}$, not all among the main features of range $\mathrm{C}$ can be predicted by utilizing the planewave analysis. The different angular locations of the maxima in $U$ and $L$ cases might be connected with the substantially different contributions of different angular components, while the strength of coupling can strongly depend on the illumination side.

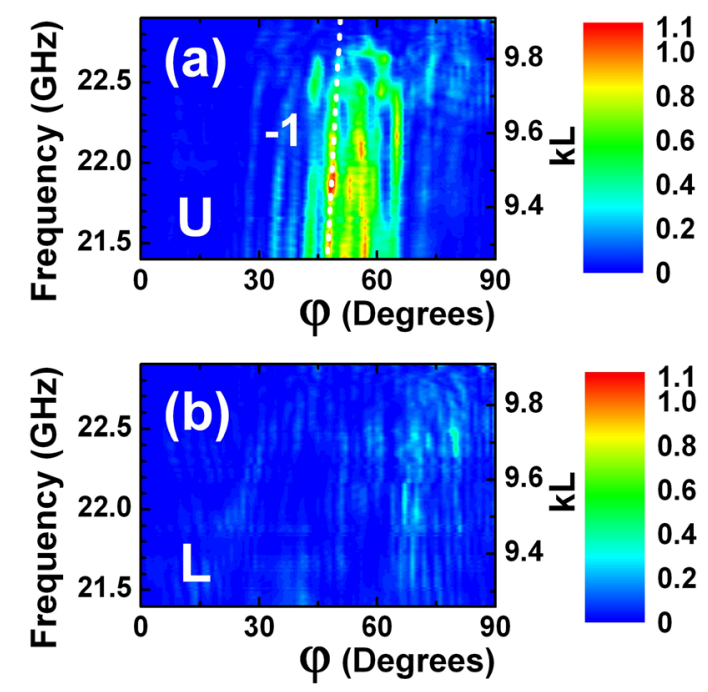

FIG. 5. The maps of the transmitted electric field intensity in $U$-case (a) and $L$-case (b); TE polarization; the white dotted line in plot (a) corresponds to $\varphi=\varphi_{g}=\pi / 2+\phi_{-1}$ obtained by using the grating formula.
The unidirectional splitting similar to that demonstrated in Figs. 3 and 4(a) can also be obtained for TE-polarized waves. Figure 5 presents the measured electric field intensity in the frequency range, where $\tau_{\Sigma}$ is nonzero in a wide range of $\varphi$ variation, while $\tau_{\Sigma}^{\leftarrow} \approx 0$. Due to the symmetry wrt to $\varphi=90^{\circ}$ line, only a half of the entire graph is presented. Comparing Figs. 3 and 5, one can see that the dual-band unidirectional splitting can be obtained. One of the two bands corresponds to TM-polarization, being identical to range $\mathrm{A}$ in Fig. 3. The second one corresponds to TE-polarization, being located at $21.4 \mathrm{GHz} \leq f \leq 22.9 \mathrm{GHz}$. At a proper parameter adjustment, another regime can be realized, in which $\tau_{\Sigma}^{\overrightarrow{ }}=\tau_{-1}^{\overrightarrow{ }}+\tau_{+1}^{\overrightarrow{1}} \neq 0$ and $\tau_{\Sigma}^{\leftarrow} \approx 0$ for both TM and TE polarization in the same frequency range.

To summarize, the possibility of combining diode and splitter functions in one PC based diode-like reciprocal device, which is connected to the regime of the broadband unidirectional splitting, has been theoretically predicted and verified in the microwave experiment. This regime appears due to merging the effects of the dispersion of FB modes and strongly asymmetric diffractions and coupling. A bandwidth larger than $15 \%$ can be realized, while the intensities of each of the two forward-transmitted narrow beams exceed the half of that of the incident beam generated by a horn antenna. At the same time, backward transmission can vanish in the entire range of the observation angle variation. Utilizing both TM and TE polarizations enables such a dual-band operation regime. In another broadband splitting regime, a strong forward transmission and a vanishing backward transmission occur in a finite but not entire range of the observation angle variation. The results obtained at the microwave frequencies indicate a route to similar performances and operation regimes at acoustic, terahertz, and optical frequencies.

This work was supported by the projects DPT-HAMIT, ESF-EPIGRAT, and NATO-SET-181 and by TUBITAK under Project Nos. 107A004, 109A015, and 109E301. A.E.S. thanks DFG for support of this work under Project No. SE1409/2-2. E.O. acknowledges partial support from the Turkish Academy of Sciences.

${ }^{1}$ A. E. Serebryannikov, Phys. Rev. B 80, 155117 (2009).

${ }^{2}$ A. O. Cakmak, E. Colak, A. E. Serebryannikov, and E. Ozbay, Opt. Express 18, 22283 (2010).

${ }^{3}$ A. E. Serebryannikov, A. O. Cakmak, and E. Ozbay, Opt. Express 20, 14980 (2012).

${ }^{4}$ S. Xu, C. Qiu, and Z. Liu, J. Appl. Phys. 111, 094505 (2012). 
${ }^{5}$ S. Feng, C. Ren, W. Wang, and Y. Wang, EPL 97, 64001 (2012).

${ }^{6}$ C. Menzel, C. Helgert, C. Rockstuhl, E.-B. Kley, A. Tünnermann, T. Pertsch, and F. Lederer, Phys. Rev. Lett. 104, 253902 (2010).

${ }^{7}$ M. Mutlu, A. E. Akosman, A. E. Serebryannikov, and E. Ozbay, Phys. Rev. Lett. 108, 213905 (2012).

${ }^{8}$ A. E. Serebryannikov, T. Magath, and K. Schuenemann, Phys. Rev. E 74, 066607 (2006).

${ }^{9}$ A. Mandatori, M. Bertolotti, and C. Sibilla, J. Opt. Soc. Am. B 24, 685 (2007).

${ }^{10}$ X.-F. Li, X. Ni, L. Feng, M.-H. Lu, C. He, and Y.-F. Chen, Phys. Rev. Lett. 106, 084301 (2011).
${ }^{11}$ X.-B. Kang, W. Tan, Z.-S. Wang, Z.-G. Wang, and H. Chen, Chin. Phys. Lett. 27, 074204 (2010).

${ }^{12}$ A. Cicek, A. O. Kaya, and B. Ulug, Appl. Phys. Lett. 100, 111905 (2012). ${ }^{13}$ C. Wang, C. Z. Zhou, and Z. Y. Li, Opt. Express 19, 26948 (2011).

${ }^{14}$ J. H. Oh, H. W. Kim, P. S. Ma, H. M. Seung, and Y. Y. Kim, Appl. Phys. Lett. 100, 213503 (2012).

${ }^{15}$ A. E. Serebryannikov, A. Y. Petrov, and E. Ozbay, Appl. Phys. Lett. 94, 181101 (2009).

${ }^{16}$ C. Luo, S. G. Johnson, J. D. Joannopoulos, and J. B. Pendry, Phys. Rev. B 65, 201104 (2002). 